\title{
Awareness and Attitude Towards Opioid and Stimulant Use and Lifetime Prevalence of the Drugs: A Study in 5 Large Cities of Iran
}

\author{
Elham Mohebbi $^{\circledR}$, Ali Akbar Haghdoost ${ }^{(}{ }^{\circledR}$, Alireza Noroozi $^{3,4}$, Hossein Molavi Vardanjani ${ }^{5}$, Ahmad Hajebi $^{6}$, \\ Roya Nikbakht ${ }^{2}$, Maryam Mehrabi ${ }^{7,8}$, Akram Jabbarinejad Kermani ${ }^{9}$, Mahshid Salemianpour ${ }^{2}$, Mohammad \\ Reza Baneshi ${ }^{*}$ (D)
}

\begin{abstract}
Background: Providing population-based data on awareness, attitude and practice of drug and stimulant use has policy implications. A national study was conducted among Iranian general population to explore life time prevalence, awareness and attitudes toward opioids and stimulant use.

Methods: We recruited subjects from 5 provinces with heterogenic pattern of drug use. Participants were selected using stratified multistage cluster sampling. Data were collected using a validated self-administered questionnaire. Logistic regression model was applied to identify the variables that are associated with drug and stimulant use.

Results: In total 2065 respondents including 1155 men $(33.96 \pm 10.40$ years old) and 910 women (35.45 \pm 12.21 years old) were recruited. Two-third of respondents had good awareness about adverse effects of opioid use. Corresponding figure in terms of stimulants was $81.4 \%$. Almost $95 \%$ of participants reported a negative attitude towards either opioid or stimulant use. The lifetime prevalence of opioid use and stimulant use were 12.9\% (men: $21.5 \%$, women: $4.0 \%$ ) and 7.3\% (men: $9.6 \%$, women: $4.9 \%$ ), respectively. Gender (adjusted odds ratio $\left.[\mathrm{AOR}]_{\mathrm{M} / \mathrm{W}}=6.92 ; 95 \% \mathrm{CI}: 2.92,16.42\right)$, education $\left(\mathrm{AOR}_{\text {undergraduate/diploma or less }}=0.49 ; 95 \% \mathrm{CI}: 0.26,0.90\right)$, and marital status ( $\left.\mathrm{AOR}_{\text {others/single }}=2.13 ; 95 \% \mathrm{CI}: 1.36,3.33\right)$ were significantly related with opioid use. With respect to stimulant use, age was negatively associated with the outcome $\left(\mathrm{AOR}_{60+120-29 \text { years }}=0.08: 95 \% \mathrm{CI} ; 0.01,0.98\right)$ and men were 2 times more likely than women to use stimulants $\left(\mathrm{OR}_{\mathrm{M} / \mathrm{w}}=2.15: 95 \%\right.$ CI: 0.83, 5.56). In addition, marital status $\left(\mathrm{AOR}_{\text {Others/singles }}=3.45 ; 95 \% \mathrm{CI}: 1.09,10.93\right)$, and awareness $\left(\mathrm{AOR}_{\text {Weak and moderate/good }}=0.40 ; 95 \% \mathrm{CI}\right.$ : $0.25,0.61$ ) were independently correlated with stimulants use.

Conclusion: While the attitude of Iranian adults toward opioid and stimulant use was negative, their awareness was not that adequate to prevent the drug use. Men and those with lower socio-economic status (SES) should be the focus of health promotion programs regarding opioid use. However, regarding stimulants use, promotion programs should target younger age groups and those with higher SES status.

Keyword: Cognition, Attitude, Prevalence, Opioid Related Disorders, Amphetamines

Copyright: $\odot 2019$ The Author(s); Published by Kerman University of Medical Sciences. This is an open-access article distributed under the terms of the Creative Commons Attribution License (http://creativecommons.org/ licenses/by/4.0), which permits unrestricted use, distribution, and reproduction in any medium, provided the original work is properly cited.

Citation: Mohebbi E, Haghdoost AA, Noroozi A, et al. Awareness and attitude towards opioid and stimulant use and lifetime prevalence of the drugs: a study of 5 large cities of Iran. Int J Health Policy Manag. 2019;8(4):222232. doi:10.15171/ijhpm.2018.128
\end{abstract}

Article History:

Received: 26 November 2017 Accepted: 15 December 2018 ePublished: 31 December 2018

*Correspondence to: Mohammad Reza Baneshi Email: rbaneshi2@gmail.com

\section{Key Messages}

Implications for policy makers

- The life time prevalence of opioids and stimulants were $12.9 \%$ (men: $21.5 \%$, women: $4.0 \%$ ) and 7.3\% (men: $9.6 \%$, women: $4.9 \%$ ) respectively.

- In terms of opioid use, health promotion programs should target men and populations with lower socio-economic status (SES) status.

- Regarding stimulants use, promotion programs should target the younger age groups and those with high SES status.

Implications for the public

The life time prevalence of opioid and stimulants use among men was 5 and 2 times higher than that of women. Although most Iranians were aware of adverse effects of opioid and stimulants use, the life time prevalence of these outcomes were high. Appropriate health promotion programs should be designed to decrease use of these drugs. 


\section{Introduction}

Illicit drug use poses striking challenges to health systems worldwide. In 2016, 275 million of world population aged 15-64 used an illicit drug, which was accounted for 5.6\% of the population. ${ }^{1}$ In addition to that, global mortality and morbidity attributed to illicit drug use, which mostly happened in Eastern Europe and Central Asia, is substantial (37926000 million disability-adjusted life years [DALYs]). Opioid dependence was the largest contributor of years life lost (YLLs) to premature mortality for illicit drug use disorders. ${ }^{2}$ In 2010, global burden of diseases estimated 804.5 and 227 for DALYs and 351.8 and 24.8 for YLLs in Iranian men and women, respectively. ${ }^{3}$

Several studies confirmed that increase in drug use disorders are associated with increase in number of shattered families, as well as crime, violence and insecurity, and health related problems including HIV and hepatitis C (via injecting drugs). ${ }^{4,5}$ It also affects economic costs, especially in low- and middle-income countries (LMICs). ${ }^{6}$

Iran is an Islamic middle-income country with a population of more than 80 million, of whom more than $70 \%$ live in urban settings. ${ }^{7}$ Except for medical purposes, using opioids and stimulants are illegal in Iran. Despite negative attitudes toward drug use among older Iranians, it has been less stigmatized and even partly prestigious among youths. ${ }^{8-10}$ This could make drug-related issues uncontrollable especially among younger adults and women. ${ }^{6}$ According to the national household survey, last year prevalence of any illicit drug use disorder except alcohol was $2.44 \%$ (1.56\% drug dependence), accounting for approximately 1.12 million Iranians (1564 years). Amongst the illicit drugs, opioids were the most prevalent one with prevalence of $2.23 \%$ (95\% CI; $1.83 \%$, $2.62 \%)$, following by cannabis $(0.56 \%)$ and amphetaminetype stimulants (ATS) (0.39\%). ${ }^{8}$ Moreover, prevalence of ATS used is very high in specific sub-population, for instance $73.9 \%$ among transit driver and $46.5 \% .^{11,12}$

Opioids are the most popular drug of use in Iran drug scene and have been used more than 5 centuries, partly due to long and porous border with Afghanistan, the main producer of illicit opioids in the world. ${ }^{2,13}$ In contrast, ATS are almost the new brand drugs in Iran's market (since 2005), ${ }^{14}$ firstly, imported from southeastern border. While ATS use is associated with many mental (insomnia, anxiety, and psychosis) and physical health complications (increased body temperature, and increased rapid heart rate), knowledge about its side effects is low. ${ }^{15-17}$ Besides, international sanctions negatively affect economic growth which might result in social harms including illicit drug use. ${ }^{18}$

There are several references to opium - "Tariak" in Persian - in Iranian medieval and classic poems where opium was considered as an "antidote" to treat diseases. Furthermore, opium has been used to treat pain, cough and diarrhea in Iranian traditional medicine. ${ }^{19}$ Nowadays, patients adhere to the faulty superstition about opium and sometimes initiating to use it by disease development without any awareness about the side effects and addictive properties. ${ }^{17,20}$ Available evidences on awareness and attitude toward opium are slim and the focuses of majority of such studies are on nursing and medical students, or high school students. ${ }^{21-23}$

Although ATS are the new brand drug in Iran illicit drug markets, a number of studies - including Persian and English and gray literature - evaluated amphetamine and ecstasy use awareness and attitude. Nonetheless, results of the most recent knowledge, attitude and practice study on Iranian youths (4868 participants aged 15-29 years) showed that $42 \%$ of participants had low knowledge, and about one-third of them had positive attitudes towards amphetamine use. ${ }^{24}$

As mentioned above majority of studies were concentrated on specific groups. However, we believe that general population should be the main target group for health education and promotion strategies. Therefore, a large study was conducted to explore life time prevalence of opioid and stimulant use, as well as awareness and attitudes toward opioid and stimulant use among Iranian adults.

\section{Methods}

\section{Participants and Sampling Design}

In 2013, Iranian National Network Scale Up (NSU) study was designed to estimate the prevalence of opioids and to stratify the country into low, intermediate, and high-risk zones. At the first step, results of that study were used to select 5 provinces: West Azerbaijan and Markazi from low risk stratum, Tehran and Khorasan-e-Razavi from moderate risk stratum, and Kerman from high risk stratum. Data are collected in capitals. Each city was stratified into low, middle and high socioeconomic zones.

A street-based consecutive sampling was used to recruit the respondents at different times and locations. The inclusion criteria were consent verbally obtained, over 18 years old, and able to reading and writing in Persian.

Sample size calculation was based on information obtained from evaluating drug abuse awareness among Afghans $(88.6 \%) .{ }^{25}$ We assumed that proportion of awareness about drug use 0.9 and $\mathrm{d}=0.03$ in both sexes $\left(n=\frac{z^{2} p(1-p)}{d^{2}}\right)$. This suggests a sample size of 400 for each province.

\section{Designing Instrument}

A self-administered questionnaire was designed considering socio-cultural context of Iran. The questionnaire was developed in 4 phases; (1) literature review, (2) expert panel review, (3) focus-group testing and evaluation, and (4) and reliability and validity assessment.

An extended literature review was conducted to ascertain common awareness and attitude statements related to opioid and stimulant use, and to find available instruments. We found various studies and reports. ${ }^{26-33}$ Reviewing the questionnaires, a question pool inquiring awareness, attitude and behavior on opioid and stimulants use was developed and discussed in expert panel reviews.

\section{Expert Panel Review}

Experts from Iranian National Center for Addiction Studies (INCAS) [Tehran, Iran], Tehran University of Medical Sciences (TUMS) [Tehran, Iran], and Neuroscience Research Center, Institute of Neuropharmacology [Kerman, Iran] reviewed the questionnaires and their invaluable feedbacks 
were gathered.

\section{Focus Group Discussion}

We conducted 2 focus group discussions (FGDs) to explore attitudes and opinions of youth population with respect to common type of drugs used among Iranian adults. A convenient sample of university students $(n=15)$ were selected from Kerman University of Medical Sciences (KUMS), Kerman, Iran. A letter was sent to these students explaining the purpose of the focus groups and assuring them that confidentiality would be maintained. In the first session, attitudes, perceived reasons and contexts of drug use initiation were discussed. In the second session the participants' opinions regarding most prevalent type of drugs were explored and then participants were asked to talk about their awareness, attitude and experiences about drug use. The focus groups were facilitated by EM.

\section{Designing of the Questionnaire}

Based on lessons from FGD, 6 conceptual frameworks were drawn for awareness, attitude and life time prevalence of opium and stimulant use. Draft was revised several times according to the experts' feedback so that the face validity of final version was approved by experts and consultants.

\section{Assessment of Reliability and Validity}

To address the validity, we asked 34 medical doctors and nurses, who worked in medical centers, to fill the questionnaire. Cronbach a was calculated at 0.90 for awareness, $83 \%$ for attitude, and $87 \%$ for practice.

For validity assessment, we asked 5 experts to describe the relevance degree of the questions (completely relevant, relatively relevant, relevant, and irrelevant). Content validity index (CVI) was estimated at about 0.8 which confirmed that validity of our questionnaire.

\section{Structure of Questionnaire}

In the first part of the questionnaire, using 20 questions with 3 possible answers (correct, incorrect, and I do not know), awareness towards opioid and stimulants was assessed. Based on the number of correct replies, 3 categories were defined, weak ( 0 to 4 ), moderate ( 5 to 7 ), and good awareness ( 8 to 10 ).

In the second section, which was consisted of 24 fivepoint Likert scale questions, we measured the attitude. The questions were associated with attitude toward social, cultural, legalization, and religious contexts. An overall attitude was determined by aggregating all positive responses ranging from -24 to +24 . Four categories were defined as: (I) strongly disagree [-24 to -13]; (II) disagree [-12 to -1]; (III) agree [+1 to +12$]$; and (IV) strongly agree $[+13$ to +24$]$.

The third part was devoted to practice of opioids and stimulants. We asked participants "Have you ever used opioid or stimulant, even one episode, during your life?" Opioids included opium, opium dross, condensed opium, heroin, crack-heroin, methadone, tramadol, and codeine. Stimulants included crystal methamphetamine, amphetamine, ecstasy, cocaine and Ritalin. Examples of drugs in each class and their common street names were provided in the questionnaire. In the last part of the questionnaire, demographic information was collected.

\section{Data Collection Methods and Instrument}

Data were collected by using the self-administered questionnaire along with guidance provided by interviewers in 2015. At the first step, each city was stratified into low, middle and high socio-economic zones. A street-based consecutive sampling was used to recruit the respondents adopting time location sampling (TLS). We randomly selected some streets at different zones and asked data collectors to collect the data at different times. We asked them to recruit participants with different clothing and appearance to maximize the generalizability. Only pedestrians who walked alone were approached.

After taking verbal consent, interviewees were asked to complete study questionnaire anonymously in the street corners. We matched the gender of data collector and interviewee. Interviewer took about 2-3 m distance from the respondent to avoid violation of the participants' privacy. However, study participants' questions were answered by the interviewers. An opaque envelope was provided for putting the questionnaire among completed questionnaires by other participants.

\section{Data Manipulation and Statistical Analysis}

Data was cleaned and prepared based on the study protocol. Variables with more than $40 \%$ missing data were excluded. For the rest of variables, missing data were imputed applying multiple imputation by chained equations (MICE) method 10 times. Linear, logistic, and multinomial regression models were used to impute missing data for linear, binary, and multinomial variables.

Chi-square test and independent samples $t$ test were used to determine the associations between qualitative and quantitative variables with opioid and stimulants use, respectively. $P$ values less than $5 \%$ were considered as statistically significant. Logistic regression was applied to assess the association of demographic characteristics, awareness, and attitude with life time prevalence of opioid and stimulant use. Appropriate weights were applied, with respect to age and gender, to adjust for our sampling scheme. All variables were offered to the model. Using each imputed data set, a logistic regression model was fitted. Results of 10 models were combined applying Rubin Rules. The variable with the highest $P$ value was excluded. The process continued until all $P$ values remained below 0.05 . This iterative process guarantees exclusion of unimportant and correlated variables. We entered variables in the model that had missing data less than $40 \%$ and backward model was performed. Analyses were done using Stata v.12 (Stata Corporation College Station, TX, USA) and R software.

\section{Results}

The response rate was $86.0 \%$. A total of 2065 individuals (1155 men and 910 women) filled the questionnaire. The mean age of men and women were $33.96 \pm 10.40$ and 35.45 \pm 12.21 respectively. More than $70 \%$ of participants had an 
Table 1. Demographic and Socioeconomic Characteristics of Participants

\begin{tabular}{|c|c|c|c|c|}
\hline Variable & Men $(n=1155)$ & Women $(n=910)$ & $P$ & Total $(\mathrm{N}=2065)$ \\
\hline Age (y) & $33.96 \pm 10.40$ & $35.45 \pm 12.21$ & .004 & $34.80 \pm 11.47$ \\
\hline \multicolumn{5}{|l|}{ Marital status, No. (\%) } \\
\hline Never married & $419(36.3)$ & $413(45.4)$ & \multirow{3}{*}{$<.0001$} & $832(40.3)$ \\
\hline Married & $644(55.8)$ & $392(43.1)$ & & $1036(50.2)$ \\
\hline Others & $92(8.0)$ & $105(11.5)$ & & $197(9.5)$ \\
\hline \multicolumn{5}{|l|}{ Education, No. (\%) } \\
\hline High school or less & $292(25.3)$ & $212(23.3)$ & \multirow{3}{*}{.395} & $504(24.4)$ \\
\hline Undergraduate & $813(70.4)$ & $650(71.4)$ & & $1463(70.8)$ \\
\hline Graduate & $50(4.3)$ & $48(5.3)$ & & $98(4.7)$ \\
\hline \multicolumn{5}{|l|}{ Income (US\$), No. (\%) } \\
\hline Less than 167 & $304(26.3)$ & 418 (45.9) & \multirow{3}{*}{$<.0001$} & $772(35.0)$ \\
\hline $167-334$ & $358(31.0)$ & $287(31.5)$ & & $645(31.2)$ \\
\hline $334-668$ & $399(34.5)$ & $173(19.0)$ & & $572(27.7)$ \\
\hline \multicolumn{5}{|c|}{ Employment status, No. (\%) } \\
\hline Unemployed & $335(29.0)$ & $554(60.9)$ & \multirow[t]{2}{*}{$<.0001$} & $889(43.1)$ \\
\hline Employed & $820(71.0)$ & $356(39.1)$ & & 1176 (56.9) \\
\hline
\end{tabular}

undergraduate academic degree. Self-reported monthly income for almost $65 \%$ of participants was less than $\$ 334$ (exchange rate $1 \$: 4200 \mathrm{IRR}$ ) (Table 1).

\section{Awareness}

Opioid Use

Most of participants had good awareness about adverse effects of opioids on health $(66.94 \%, 95 \%$ CI: $54.4,79.5)$ and only $1.5 \%$ were not aware of adverse effects (95\% CI: 0.0 , 1.5). About one-third of respondents had moderate level of awareness (31.5\%, 95\% CI: 20.4, 42.6).

Higher education was positively correlated with higher awareness. While 75\% (95\% CI: $69.5,80.9)$ of those with graduate degree had good awareness, corresponding figure among those with high school or lower degree was $60.1 \%$ (95\% CI: 38.1, 82.1). Marital status $(P=.09)$ and employment status $(P=.58)$ and age $(P=.17)$ of respondents did not show association with their awareness but income $(P<.0001)$ (Table 2).

\section{Stimulant use}

Almost 81.4\% (95\% CI: 76.7, 86.2) of participants had good awareness about adverse effects of stimulants on health and only 3\% (95\% CI: 1.2, 4.8) had weak awareness (Table 3).

Table 2. Awareness of Iranian Adults Towards Opioid Use, 2015

\begin{tabular}{|c|c|c|c|c|c|c|}
\hline & \multirow{2}{*}{ Variable } & & \multicolumn{4}{|c|}{ Knowledge $(\%, 95 \% \mathrm{Cl})$} \\
\hline & & & Good & Moderate & Weak & $P$ \\
\hline \multirow{20}{*}{ Opioids } & \multirow{2}{*}{ Gender } & Men & $63.3(49.0,77.7)$ & $35.0(22.4,47.6)$ & $1.6(0.0,3.5)$ & \multirow{2}{*}{$<.0001$} \\
\hline & & Women & $70.8(50.2,91.2)$ & $27.9(9.6,46.2)$ & $1.4(0.0,3.6)$ & \\
\hline & \multirow{3}{*}{ Education } & Diploma or less & $60.1(38.1,82.1)$ & $37.6(16.6,58.6)$ & $2.2(0.0,3.8)$ & \multirow{3}{*}{$<.0001$} \\
\hline & & Undergraduate & $69.0(61.7,76.3)$ & $29.6(23.6,35.6)$ & $1.3(0.0,2.9)$ & \\
\hline & & Graduate & $75.2(69.5,80.9)$ & $24.5(19.1,30.5)$ & - & \\
\hline & \multirow{5}{*}{ Age } & $20-29$ & $66.3(54.4,78.2)$ & $32.6(22.1,43.0)$ & $1.1(0.0,2.6)$ & \multirow{5}{*}{.165} \\
\hline & & $30-39$ & $65.4(55.6,75.2)$ & $32.9(25.0,40.8)$ & $1.7(0.0,3.7)$ & \\
\hline & & $40-49$ & $67.2(51.4,82.9)$ & $30.8(16.7,45.0)$ & $2.0(0.0,4.7)$ & \\
\hline & & $50-59$ & $70.8(52.1,82.9)$ & $26.8(8.7,45.0)$ & $2.4(0.0,4.6)$ & \\
\hline & & More than 60 & $69.9(48.0,91.8)$ & $30.0(8.2,52.0)$ & - & \\
\hline & \multirow{4}{*}{ Income } & Less than 167 & $60.8(42.0,79.6)$ & $37.0(19.6,54.3)$ & $2.2(0.0,3.9)$ & \multirow{4}{*}{$<.0001$} \\
\hline & & $167-334$ & $68.3(60.5,76.2)$ & $30.0(24.2,35.9)$ & $1.6(0.0,3.9)$ & \\
\hline & & $334-668$ & $74.2(64.3,84.0)$ & $25.4(16.1,34.8)$ & $0.3(0.0,1.1)$ & \\
\hline & & More than 668 & $68.7(63.9,73.5)$ & $30.0(24.9,35.3)$ & $1.2(0.0,2.7)$ & \\
\hline & \multirow{3}{*}{ Marital status } & Never married & $68.6(57.6,79.6)$ & $30.1(19.9,40.4)$ & $1.2(0.0,2.1)$ & \multirow{3}{*}{.085} \\
\hline & & Married & $67.7(53.1,82.3)$ & $30.5(17.7,43.3)$ & $1.7(0.0,3.7)$ & \\
\hline & & Others & $60.0(45.4,74.5)$ & $38.6(25.5,51.7)$ & $1.4(0.0,3.7)$ & \\
\hline & \multirow{2}{*}{ Employment status } & Unemployed & $65.5(45.0,86.1)$ & $33.1(14.1,52.0)$ & $1.4(0.0,3.0)$ & \multirow{2}{*}{.579} \\
\hline & & Employed & $68.2(62.7,73.7)$ & $30.2(25.9,34.4)$ & $1.6(0.0,3.2)$ & \\
\hline & Total & & $66.94(54.4,79.5)$ & $31.5(20.4,42.6)$ & $1.5(0.0,3.0)$ & \\
\hline
\end{tabular}


Table 3. Awareness of Iranian Adults Towards Stimulant Use, 2015

\begin{tabular}{|c|c|c|c|c|c|c|}
\hline & \multirow{2}{*}{ Variable } & & \multicolumn{4}{|c|}{ Knowledge $(\%, 95 \% \mathrm{CI})$} \\
\hline & & & Good & Moderate & Weak & $P$ \\
\hline \multirow{20}{*}{ Stimulants } & \multirow{2}{*}{ Gender } & Men & $80.7(71.4,85.4)$ & $15.6(7.3,17.8)$ & $3.7(1.2,6.1)$ & \multirow{2}{*}{$<.0001$} \\
\hline & & Women & $82.2(79.0,85.4)$ & $15.5(13.1,17.8)$ & $2.3(0.0,4.6)$ & \\
\hline & \multirow{3}{*}{ Education } & Diploma or less & $73.7(68.1,79.4)$ & $21.2(13.6,28.7)$ & $5.1(2.3,7.8)$ & \multirow{3}{*}{$<.0001$} \\
\hline & & Undergraduate & $83.8(76.9,90.8)$ & $13.8(7.5,20.0)$ & $2.4(0.0,3.8)$ & \\
\hline & & Graduate & $90.3(85.1,95.5)$ & $9.2(4.8,13.8)$ & $3.8(0.0,1.4)$ & \\
\hline & \multirow{5}{*}{ Age } & $20-29$ & $80.5(75.1,85.9)$ & $16.7(11.3,22.0)$ & $2.8(1.6,4.0)$ & \multirow{5}{*}{.006} \\
\hline & & $30-39$ & $83.1(77.6,88.6)$ & $12.9(7.4,18.4)$ & $4.0(0.0,7.5)$ & \\
\hline & & $40-49$ & $79.5(71.8,87.0)$ & $16.9(11.3,22.4)$ & $3.7(0.0,7.2)$ & \\
\hline & & $50-59$ & $83.2(77.2,89.3)$ & $15.6(10.1,21.0)$ & $1.1(0.0,3.0)$ & \\
\hline & & More than 60 & $81.9(58.2,100)$ & $16.8(0.0,41.4)$ & $1.2(0.0,2.8)$ & \\
\hline & \multirow{4}{*}{ Income } & Less than 167 & $79.0(76.0,82.0)$ & $15.7(12.2,19.2)$ & $5.3(2.8,7.8)$ & \multirow{4}{*}{.0009} \\
\hline & & $167-334$ & $81.5(76.1,86.9)$ & $16.0(12.0,20.0)$ & $2.5(0.0,4.6)$ & \\
\hline & & $334-668$ & $82.1(72.2,91.9)$ & $17.0(7.6,26.4)$ & $0.9(0.0,2.3)$ & \\
\hline & & More than 668 & $91.0(82.5,99.5)$ & $8.5(0.0,16.5)$ & $0.4(0.0,1.5)$ & \\
\hline & \multirow{3}{*}{ Marital status } & Never married & $81.3(74.7,87.9)$ & $15.8(8.7,22.9)$ & $2.8(1.4,4.2)$ & \multirow{3}{*}{0.028} \\
\hline & & Married & $82.8(77.0,88.6)$ & $14.2(9.3,19.0)$ & $3.0(0.0,6.2)$ & \\
\hline & & Others & $76.7(68.9,84.4)$ & $19.8(9.8,29.9)$ & $3.4(0.0,6.5)$ & \\
\hline & \multirow{2}{*}{ Employment status } & Unemployed & $81.4(76.7,86.2)$ & $15.1(10.8,19.3)$ & $3.5(1.8,5.1)$ & \multirow{2}{*}{.888} \\
\hline & & Employed & $81.4(76.2,86.7)$ & $16.0(10.7,21.3)$ & $2.5(0.0,4.9)$ & \\
\hline & Total & & $81.4(76.7,86.2)$ & $15.5(11.2,20.0)$ & $3.0(1.2,4.8)$ & \\
\hline
\end{tabular}

Higher education $(P<.0001)$ and monthly income $(P=.009)$ were positively correlated with higher awareness. There was association between gender and awareness $(P<.0001)$, age $(P=.006)$, and marital status $(P=.028)$ with outcome. Employment status $(P=.888)$ showed no association with respondents' awareness (Table 3 ).

\section{Attitude}

Opioid Use

95.5\% (95\% CI: 91.5, 99.5) of participants reported a negative attitude toward opioid use. Higher education was the only variable which was significantly correlated with attitude $(P=.02)$ (Table 4$)$. While $7.4 \%$ of respondents with low education reported a positive attitude, corresponding figure among graduated respondents was $1.2 \%$.

\section{Stimulant Use}

More than $96.0 \%$ (95\% CI: 93.7, 99.5) of respondents had a negative attitude towards stimulants. Almost all of respondents (99.6, 95\% CI: 98.5, 100) with a graduate degree reported a negative attitude regarding stimulants consumption. Negative attitude among less educated respondents was 95.1 (95\% CI: 92.8, 97.4; $P=.05)$. Marital status was marginally associated with attitude $(P=.06)$. Negative attitude in men and women were estimated at $97.1 \%$ and $96.2 \%$ respectively $(P=.72)$. Age $(P=.32)$, monthly income $(P=.19)$ and employment status $(P=.11)$ had no significant association with attitude toward stimulants use (Table 4).

\section{Life Time Prevalence \\ Opioid Use}

The self-reported life time prevalence of opioid use was estimated at $12.9 \%(95 \%$ CI: 6.8, 19.0) (Table 5). Lifetime prevalence of opioid use was significantly higher in men than women $-21.5 \%$ (95\% CI: 18.7, 24.3) versus $4.0 \%$ (95\% CI: $1.9,6.1)$. Prevalence in those aged $>60$ and $40-49$ were 20.9\% (95\% CI: 0.0, 43.4\%), and 19.3\% (95\% CI: 8.8, 29.7) respectively. With respect to the marital status, the highest prevalence was estimated for whom categorized as "others," including divorced, widows and separated (20.5, 95\% CI: $10.1,30.9)$. Respondents with lowest education (19.8\%, 95\% CI: $5.0,34.5)$ and who were employed (15.7\% 95\% CI: 9.7, 21.8) reported highest lifetime prevalence of opioid use. With respect to income, highest prevalence was observed among middle lower incomes (\$167-\$334 per month) (Table 5).

\section{Stimulant Use}

Overall lifetime prevalence of stimulant use was estimated at 7.3\% (95\% CI: 5.2, 9.4). Estimated prevalence for men was about 2 times that of women - 9.6\% (95\% CI: 6.1, 13.1) versus $4.9 \%$ (95\% CI: 0.7, 9.2). Among different age groups, the highest lifetime prevalence was observed among 20-29 years, $12.0 \%$ (95\% CI: 7.8, 16.2). As the respondents were getting older, the prevalence was sharply declined to 1.7 (95\% CI: $0.0,5.3)$. With respect to marital status, those categorized as "others" reported the highest prevalence of lifetime stimulant use $(13.6,95 \%$ CI: 5.6, 21.6). Those with graduate degree were about 2 times higher than that of those with a degree below diploma (11.9 versus 6.5). Prevalence of stimulant use among those felt into highest monthly income category was noticeable (10.5, 95\% CI: 7.0, 14.0) (Table 6).

\section{Correlates of Lifetime Prevalence Opioid Use}

Results of multivariable logistic regression modeling suggested that gender $\left(\mathrm{AOR}_{\mathrm{M} / \mathrm{F}}=6.92 ; 95 \% \mathrm{CI}: 2.92,16.41\right)$, 
Table 4. Attitude of Iranian Adults Towards Opioid and Stimulant Use, 2015

\begin{tabular}{|c|c|c|c|c|c|c|c|}
\hline \multirow{2}{*}{ Variable } & & \multicolumn{3}{|c|}{ Opioids ( $n=2065$ ) } & \multicolumn{3}{|c|}{ Stimulants $(n=2065)$} \\
\hline & & Negative & Positive & $P$ & Negative & Positive & $P$ \\
\hline \multirow{2}{*}{ Gender } & Men & $94.5(88.4,100)$ & $5.5(0.0,11.6)$ & \multirow{2}{*}{.55} & $97.1(93.0,100)$ & $2.9(0.0,7.0)$ & \multirow{2}{*}{.72} \\
\hline & Women & $96.6(91.8,100)$ & $3.4(0.0,8.2)$ & & $96.2(92.2,100)$ & $3.9(0.0,7.9)$ & \\
\hline \multirow{3}{*}{ Education } & High school or less & $92.6(88.2,97.0)$ & $7.4(3.0,11.8)$ & \multirow{3}{*}{.02} & $95.1(92.8,7.4)$ & $4.9(2.6,7.2)$ & \multirow{3}{*}{.05} \\
\hline & Undergraduate & $96.4(93.0,99.9)$ & $3.6(0.1,7.0)$ & & $96.9(93.8,100)$ & $3.1(0.0,6.2)$ & \\
\hline & Graduated & $98.8(96.0,100)$ & $1.2(0.0,4.0)$ & & $99.6(98.5,100)$ & $0.4(0.0,1.5)$ & \\
\hline \multirow{5}{*}{ Age } & $20-29$ & $95.3(0.9,99.9)$ & $4.7(0.06,9.3)$ & \multirow{5}{*}{.37} & $96.0(91.9,100)$ & $4.0(0.0,8.1)$ & \multirow{5}{*}{.32} \\
\hline & $30-39$ & $96.2(0.9,98.6)$ & $3.8(1.4,6.3)$ & & $97.0(94.8,99.3)$ & $3.0(0.7,5.2)$ & \\
\hline & $40-49$ & $96.8(91.3,100)$ & $3.2(0.0,8.7)$ & & $98.0(95.4,100)$ & $2.0(0.0,4.6)$ & \\
\hline & $50-59$ & $93.5(87.7,99.2)$ & $6.6(0.7,12.3)$ & & $95.3(91.3,99.4)$ & $4.7(0.6,8.8)$ & \\
\hline & More than 60 & $93.9(87.2,100)$ & $6.1(0.0,12.8)$ & & $96.8(92.4,100)$ & $3.2(0.0,7.6)$ & \\
\hline \multirow{4}{*}{ Income } & Less than 167 & $94.2(89.7,98.6)$ & $5.8(1.4,10.3)$ & \multirow{4}{*}{.16} & $95.8(92.8,98.9)$ & $4.2(1.2,7.2)$ & \multirow{4}{*}{.19} \\
\hline & $167-334$ & $96.4(92.7,100)$ & $3.6(0.0,7.2)$ & & $97.0(93.8,100)$ & $3.1(0.0,6.2)$ & \\
\hline & $334-668$ & $95.7(92.3,99.1)$ & $4.3(0.9,7.7)$ & & $96.7(93.8,99.5)$ & $3.3(0.5,6.2)$ & \\
\hline & More than 668 & $97.8(92.9,100)$ & $2.2(0.0,7.1)$ & & $99.0(96.5,100)$ & $1.0(0.0,3.5)$ & \\
\hline \multirow{3}{*}{ Marital status } & Never married & $96.0(92.5,99.5)$ & $4.0(0.5,7.5)$ & \multirow{3}{*}{.6} & $97.1(94.3,99.9)$ & $2.9(0.1,5.7)$ & \multirow{3}{*}{.06} \\
\hline & Married & $94.7(90.3,99.0)$ & $5.3(1.0,9.7)$ & & $96.1(93.2,98.9)$ & $3.9(1.0,6.8)$ & \\
\hline & Others & $97.4(94.3,100)$ & $2.6(0.0,5.7)$ & & $97.5(94.7,100)$ & $2.5(0.0,5.3)$ & \\
\hline \multirow{2}{*}{ Employment status } & Unemployed & $94.7(90.3,99.1)$ & $5.3(0.9,9.7)$ & \multirow{2}{*}{.24} & $95.6(92.2,99.0)$ & $4.4(1.0,99.0)$ & \multirow{2}{*}{.11} \\
\hline & Employed & $96.2(92.3,100)$ & $3.8(0.0,7.7)$ & & $97.5(94.8,100)$ & $2.5(0.0,5.2)$ & \\
\hline Total & & $95.5(91.5,99.5)$ & $4.5(0.5,8.5)$ & & $96.6(93.7,99.5)$ & $3.4(0.5,6.3)$ & \\
\hline
\end{tabular}

Table 5. Lifetime Prevalence $(95 \% \mathrm{Cl})$ of Opioid Use Amongst Iranian Adults

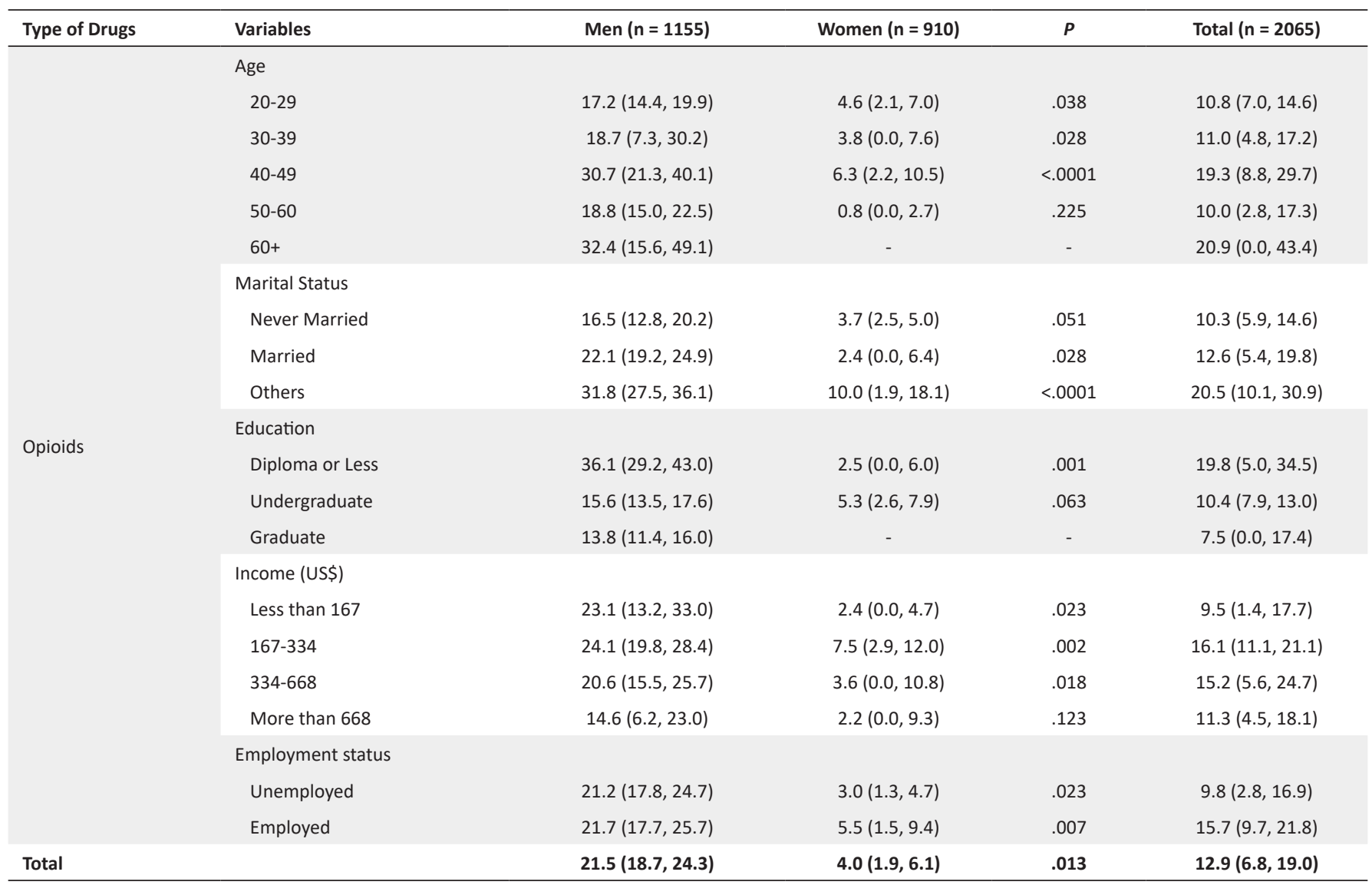


Table 6. Lifetime Prevalence $(95 \% \mathrm{Cl})$ of Stimulants Use Amongst Iranian Adults

\begin{tabular}{|c|c|c|c|c|c|}
\hline Type of Drugs & Variables & Men $(n=1155)$ & Women $(n=910)$ & $P$ & Total $(n=2065)$ \\
\hline \multirow{22}{*}{ Stimulants } & Age & & & & \\
\hline & $20-29$ & $14.7(11.4,18.1)$ & $9.4(0.0,18.8)$ & .233 & $12.0(7.8,16.2)$ \\
\hline & $30-39$ & $8.7(7.2,10.1)$ & $3.2(1.1,5.4)$ & .322 & $5.9(4.3,7.4)$ \\
\hline & $40-49$ & $8.1(4.6,11.6)$ & $2.3(0.0,4.6)$ & .349 & $5.3(3.2,7.5)$ \\
\hline & $50-60$ & $3.2(0.0,9.2)$ & $0.4(0.0,1.7)$ & .755 & $1.9(0.0,4.7)$ \\
\hline & $60+$ & $2.7(0.0,8.6)$ & - & - & $1.7(0.0,5.3)$ \\
\hline & Marital Status & & & & \\
\hline & Never Married & $13.6(10.0,17.1)$ & $4.8(2.5,7.2)$ & .109 & $9.3(7.6,10.9)$ \\
\hline & Married & $5.8(1.8,9.8)$ & $2.8(0.0,5.0)$ & .558 & $4.4(2.1,6.6)$ \\
\hline & Others & $14.5(3.3,25.6)$ & $12.8(0.0,25.8)$ & .684 & $13.6(5.6,21.6)$ \\
\hline & Education & & & & \\
\hline & Diploma or Less & $12.2(6.6,17.8)$ & $0.4(0.0,1.0)$ & .473 & $6.5(1.2,11.8)$ \\
\hline & Undergraduate & $7.5(3.8,11.2)$ & $6.5(1.9,11.1)$ & .818 & $7.0(4.3,9.7)$ \\
\hline & Graduate & $14.3(11.6,17.0)$ & $9.0(4.5,13.4)$ & .237 & $11.9(7.3,16.3)$ \\
\hline & Income (US\$) & & & & \\
\hline & Less than 167 & $15.9(12.2,19.6)$ & $2.7(0.0,5.5)$ & .077 & $7.2(2.9,11.6)$ \\
\hline & $167-334$ & $7.8(1.2,14.1)$ & $8.5(2.6,14.5)$ & .869 & $8.1(3.4,12.7)$ \\
\hline & $334-668$ & $5.5(2.0,9.0)$ & $5.8(3.2,8.4)$ & .944 & $5.6(3.0,8.2)$ \\
\hline & More than 668 & $12.1(6.5,17.7)$ & $6.0(3.2,8.8)$ & .210 & $10.5(7.0,14.0)$ \\
\hline & Employment status & & & & \\
\hline & Unemployed & $11.1(6.0,16.3)$ & $4.8(0.0,10.3)$ & .220 & $7.2(3.4,10.9)$ \\
\hline & Employed & $8.7(5.9,11.6)$ & $5.2(3.0,7.3)$ & .456 & $7.4(6.1,8.8)$ \\
\hline Total & & $9.6(6.1,13.1)$ & $4.9(0.7,9.2)$ & .335 & $7.3(5.2,9.4)$ \\
\hline
\end{tabular}

and marital status (AOR $=2.13$; 95\% CI: $1.36,3.33$ ) were significantly correlated with lifetime prevalence of opioid use (Table 7). In addition, one level increase in education was associated with $60 \%$ decrease in odds of opioids use $(P<.001)$.

\section{Stimulant Use}

One year increase in age was associated with 7\% decrease in odds of stimulants use $(P=.04)$. Marital status ( $\mathrm{AOR}_{\text {Others/singles }}$ $=3.45 ; 95 \% \mathrm{CI}: 1.09,10.93)$, and awareness $\left(\mathrm{AOR}_{\text {Weak and moderate/ }}\right.$ good $=0.40 ; 95 \%$ CI: $0.25,0.60)$ had independent correlation with life time prevalence of stimulants use (Table 8).

\section{Discussion}

A large cross-sectional study in 5 major cities of Iran was performed to estimate population-based statistics on the awareness, attitudes towards opioid and stimulant use and their lifetime prevalence. This study reflected that participants had a good level of awareness of opioid (67\%) and stimulant use $(82 \%)$ and almost all of the respondents had negative attitudes toward the drugs. Lifetime prevalence of opioid and stimulant use was $12.9 \%$ and $7.2 \%$, respectively.

Good level of awareness observed among Iranian adults in general population was higher than corresponding figures among Iranian students ${ }^{34,35}$; which may be due to lack of comprehensive and mandatory course in educational system, though lower awareness is expected among youths. Moreover, a most recent population-based study on Iranian youths (1929 years) showed that $42 \%$ of them had good knowledge on methamphetamine, which is lower than our findings. We should mention that our questioner was not the same as that used in study. ${ }^{24}$ While we covered all types of stimulants and drugs, they only asked about methamphetamines in details. Some other evidences, related to pre-epidemic phase of stimulants use in Iran, reflected very low knowledge among students(1\%-2\%). ${ }^{36,37}$ Amphetamine epidemic in Iran happened during 2005-2008 when domestic production of glass was burgeoned. During the epidemic mass media and newspapers abundantly provided documentaries, video clips and articles about adverse effects of stimulant use, therefore, in parallel these have formed negative attitude toward stimulants use. ${ }^{14,38}$

On the other hand, limited evidence on awareness about opioids among Iranian adults is available. Being on the main route of drug trafficking, from Western Asia to Eastern Europe, as well as cultural issues (medical and recreational uses) and relatively low cost, provoke Iranian to use opioids. In contrast having good knowledge about opioid use, colonialism of the Great Britain on Iran opioid market caused negative attitude toward opioid use..$^{39,40}$

While men were more likely to use opioids and stimulants, no association between gender and attitude toward opioids and stimulants use was found. This might be partially justified by previous evidences which suggested that a considerable proportion of people use in their life course drugs before the formation of negative attitudes. ${ }^{41}$

Lifetime prevalence of opioid use reported about $17 \%$ in the Northern and southeastern of Iran (ever users), which are known as hot zones and high prevalent areas. ${ }^{42,43}$ Estimation 
Table 7. Determinants of Opioid Use in Iran

\begin{tabular}{|c|c|c|c|c|c|c|}
\hline Type of Drugs & Variables & Crude OR $(95 \% \mathrm{Cl})$ & $P$ & & Adjusted OR $(95 \% \mathrm{Cl})$ & $P$ \\
\hline \multirow{30}{*}{ Opioids } & Age & & & & & \\
\hline & $20-29$ & \multicolumn{5}{|c|}{ Reference } \\
\hline & $30-39$ & $1.02(0.58,1.81)$ & .928 & & $0.97(0.46,2.03)$ & .931 \\
\hline & $40-49$ & $1.97(1.39,2.79)$ & .002 & & $1.07(1.70,2.89)$ & .037 \\
\hline & $50-59$ & $0.92(0.51,1.66)$ & .762 & & $0.88(0.49,1.59)$ & .629 \\
\hline & $60+$ & $2.19(0.72,6.60)$ & .141 & & $1.39(0.45,4.24)$ & .519 \\
\hline & Gender & & & & & \\
\hline & Men & \multicolumn{5}{|c|}{ Reference } \\
\hline & Women & $6.58(3.74,11.59)$ & $<.0001$ & & $6.92(2.92,16.42)$ & $<.0001$ \\
\hline & Education & & & & & \\
\hline & Diploma or less & \multicolumn{5}{|c|}{ Reference } \\
\hline & Undergraduate & $0.47(0.22,1.00)$ & .051 & & $0.49(0.26,0.90)$ & .027 \\
\hline & Marital status & & & & & \\
\hline & Single & \multicolumn{5}{|c|}{ Reference } \\
\hline & Married & $1.26(0.79,2,00)$ & .282 & & $1.06(0.79,1.42)$ & .663 \\
\hline & Others & $2.25(1.42,3.60)$ & .004 & & $2.13(1.36,3.33)$ & .004 \\
\hline & Income & & & & & \\
\hline & Less than 167 & \multicolumn{5}{|c|}{ Reference } \\
\hline & $167-334$ & $1.82(0.72,4.62)$ & .174 & & $1.34(0.54,3.29)$ & .760 \\
\hline & $334-668$ & $1.70(0.63,4.58)$ & 1.230 & & $1.10(0.63,1.91)$ & .400 \\
\hline & More than 668 & $1.21(0.53,2.74)$ & .550 & & $0.68(0.26,1.78)$ & .382 \\
\hline & Employment status & & & & & \\
\hline & Unemployed & \multicolumn{5}{|c|}{ Reference } \\
\hline & Employed & $1.71(0.90,3.24)$ & .089 & & $1.22(0.73,2.04)$ & .394 \\
\hline & Attitudes & & & & & \\
\hline & Positive & $1.85(0.57,6.02)$ & .263 & & $1.52(0.58,3.99)$ & .347 \\
\hline & Awareness & & & & & \\
\hline & Good & \multicolumn{5}{|c|}{ Reference } \\
\hline & Weak & $0.62(0.21,1.84)$ & .341 & & $0.61(0.88,4.23)$ & .574 \\
\hline & Moderate & $0.30(0.06,1.40)$ & .109 & & $0.34(0.03,4.00)$ & .341 \\
\hline
\end{tabular}

Abbreviation: OR, odds ratio.

of prevalence of opioid use in specific populations was of great attention. Exploring drug use among high school students indicated wide variation of experiencing opioids, $1.2 \%$ to $8.6 \%{ }^{22}$ Some specific populations such as drivers and prisoners were categorized as high risk groups of opioid use, with prevalence ranged $26.5 \%$ to $79 \%{ }^{44,45}$ As opioids are the most prevalent drug in $\operatorname{Iran}^{8}$, appropriate strategies and harm reduction programs should be designed to decrease its burden and adverse side effects such as hepatitis C and HIV/AIDS.

Though stimulants marketing is relatively new in Iran, its life time prevalence among those aged 19-29 was estimated at $6 \%{ }^{24}$ Results of a similar study in Tehran confirmed our results. ${ }^{46}$ The lifetime prevalence of stimulants varied among subpopulations, less than $1 \%$ among women to more than $13 \%$ among body builders. ${ }^{47}$

We have seen remarkable gender difference in terms of opioids and stimulants use. This finding was in line with others results. It is known that men are more likely to show high risk behaviors than women, in particular in Iran due to cultural background. ${ }^{22,46,48}$ Moreover, it has been indicated that men usually transit to drug use from cigarette smoking
- prevalence of daily smoking among men is about $24 \%$ in Iran - while women initiates with opium. ${ }^{49}$ Despite lower prevalence of opioid use among women, they need specific harm reduction programs because women often provide sexual intercourse in exchange for money or drug. This in turn increases sexual transmitted infections such as HIV/ AIDS..$^{50}$

As it had reported in previous works, ${ }^{9,51}$ more educated respondents were less likely to use opioids. It may not be considered as a result of higher awareness or more negative attitude as these were not remained into the final multivariable model.

Those who formed that category "divorced or widow or separated" reported significant higher lifetime prevalence of both of opioid and stimulant use. This population is more likely to have high risk behaviors as compared with single and married populations, partially because of mental distress which is one of the most important reason for initiating drugs. ${ }^{52,53}$ As the number of divorced or separated peoples has been increasing in recent years, ${ }^{54}$ it may be the future concern of the public health in Iran. 
Table 8. Determinants of Stimulants Use in Iran

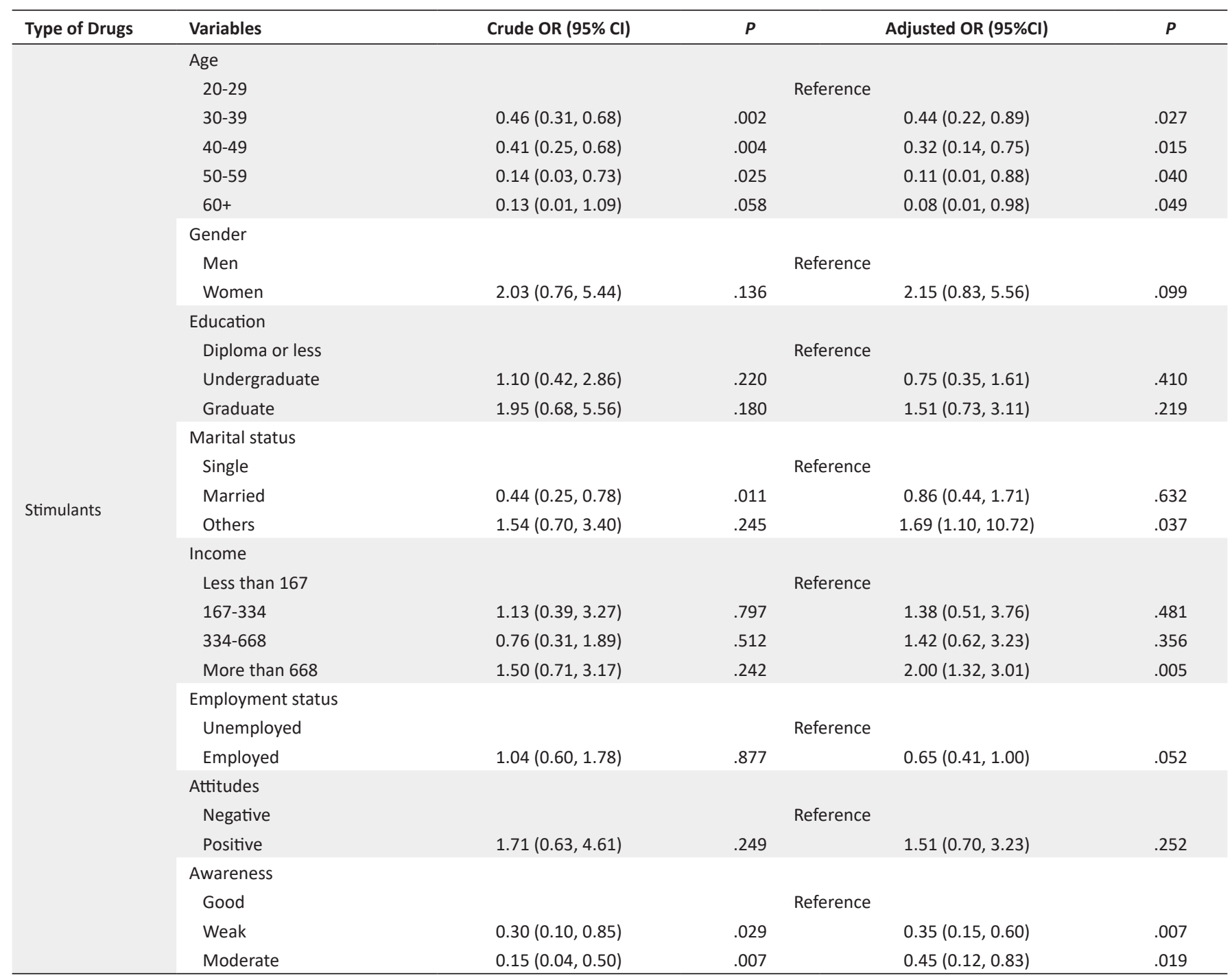

Abbreviation: OR, odds ratio.

\section{Limitations}

We were not able to estimate current prevalence of opioids and stimulants use; as questions about recent use is more sensitive.

We should also mention that we used direct question to estimate prevalence. It has been shown that direct questions prone to prestige biases and might provide an under-estimated prevalence. We already applied indirect methods such as NSU among general population. In NSU, respondents describe their network in terms of drug use. Therefore, prestige bias is of less concern. Instead recall bias and invisibility of hidden characteristics might provide inaccurate estimates.

As no standardized instrument was available, we used a researcher-developed instrument to measure awareness, attitude and life time prevalence. However, this limitation is pervasive in this field in Iran. Therefore, development of a standardized questionnaire for such studies on the drug use would be helpful for future works.

One more limitation of our study was that we were not able to conduct a household survey. We approached pedestrians in the street. Therefore, our sample was not a random sample of the general population. However, our previous experiences suggested that street-based sampling was the optimum approach in the case of sensitive characteristics in Iran. ${ }^{55,56}$

\section{Conclusion}

Despite negative attitudes of Iranian adults toward opioid and stimulant use, their awareness was less adequate to prevent high risk behavior. Men and participants with lower socio-economic status (SES) should be considered as a main target population of health promotion programs for opioid use. However, regarding stimulants use, health promotion programs which focused mostly on youths and who had higher SES could be more effective. Considering wide distribution of virtual world and increasing trend of internet access, we suggest that future studies work on sharing info-graphs of illicit drug use side effects and also assessing educational packages on the network, which could be the fastest, easiest, and most cost-effective way of informing the society. Iran education curriculum needs to be revised in which drug use should be included. In addition, parent should be trained periodically to recognize high-risk behavior warning signs in 
children and adolescents. Further investigations are needed to identify appropriate and effective approaches based on Iranian socio-cultural context that benefit the population with available resources.

\section{Acknowledgements}

This work has been supported by Iranian Ministry of Health and Medical Education, Tehran, Iran (grant number 93/428).

\section{Ethical issues}

The project was conducted according to the principles of the Helsinki declaration. The anonymity of all participants in each stage of project from focus groups to national respondents remained completely anonymous. The project was approved by Ethical Committee of Kerman University of Medical Sciences, Kerman, Iran (K/93/395).

\section{Competing interests}

Alireza Noroozi, Ahmad Hajebi, and Maryam Mehrabi are affiliated to Ministry of Health and Medical Education (MoHME), Tehran, Iran. But we confirm that there was no competing interest.

\section{Authors' contributions}

EM, AAH, ARN, AH, RN, AJK, MS, MRB, and MM contributed to the study design. RN performed statistical analyses of national data, interpretation of data, and commenting on manuscript. MRB and EM supervised the study and contributed to all parts of the paper. All authors read and approved the final version of the paper.

\section{Authors' affiliations}

${ }^{1}$ HIVISTI Surveillance Research Center, and WHO Collaborating Center for HIV Surveillance, Institute for Futures Studies in Health, Kerman University of Medical Sciences, Kerman, Iran. ${ }^{2}$ Modeling in Health Research Center, Institute for Futures Studies in Health, Kerman University of Medical Sciences, Kerman, Iran. ${ }^{3}$ School of Advanced Technologies in Medicine (SATiM), Tehran University of Medical Sciences (TUMS), Tehran, Iran. ${ }^{4}$ Iranian National Center for Addiction Studies (INCAS), Tehran University of Medical Sciences (TUMS), Tehran, Iran. ${ }^{5} \mathrm{MPH}$ Department, Medical School, Shiraz University of Medical Sciences, Shiraz, Iran. ${ }^{6}$ Research Center for Addiction \& Risky Behavior (ReCARB), Psychiatric Department, Iran University of Medical Sciences, Tehran, Iran. ${ }^{7}$ Department of Sociology, Faculty of Social Sciences and Economics, Alzahra University, Tehran, Iran. ${ }^{8}$ Department of Mental, Social Health and Drug Use, Ministry of Health, Tehran, Iran. ${ }^{9}$ Social Determinants of Health Research Center, Institute for Futures Studies in Health, Kerman University of Medical Sciences, Kerman, Iran.

\section{References}

1. Maqbool S. UNODC report documents expansion in opioid crisis, prescription drug abuse. https://www.thenews.com.pk/print/364536unodc-report-documents-expansion-in-opioid-crisis-prescriptiondrug-abuse. Published 2018.

2. Whiteford HA, Ferrari AJ, Degenhardt L, Feigin V, Vos T. The global burden of mental, neurological and substance use disorders: an analysis from the Global Burden of Disease Study 2010. PLoS One. 2015;10(2):e0116820. doi:10.1371/journal.pone.0116820

3. Moazen B, Shokoohi M, Noori A, et al. Burden of Drug and Alcohol Use Disorders in Iran: findings from the Global Burden of Disease Study 2010. Arch Iran Med. 2015;18(8):480-485.

4. Bahr SJ, Maughan SL, Marcos AC, Li B. Family, religiosity, and the risk of adolescent drug use. J Marriage Fam. 1998;60(4):979-992. doi:10.2307/353639

5. United Nations Office on Drugs and Crime (UNODC). World Drug Report 2015. Vienna: United Nations; 2012.

6. UNODC. United Nations Office on Drugs and Crime and United Nations Development Programme. Violence and Injury Prevention: Global status report on violence prevention. https://www.who. int/violence injury prevention/violence/status report/2014/en/. Accessed December 2018.

7. Iran Census 2016. Statistical Center of Iran website. https://www. amar.org.ir/english. Published 2016.

8. Amin-Esmaeili M, Rahimi-Movaghar A, Sharifi V, et al. Epidemiology of illicit drug use disorders in Iran: prevalence, correlates, comorbidity and service utilization results from the Iranian Mental Health Survey. Addiction.2016;111(10):1836-1847.doi:10.1111/add.13453

9. Mokri A. Brief overview of the status of drug abuse in Iran. Arch Iran Med. 2002;5(3):184-190.

10. Samii AW. Drug Abuse: Iran's “Thorniest Problem." Brown J World Aff. 2003;9(2):283-299.

11. Narenjiha H, Rafiey $H$, Jahani MR, Assari S, Moharamzad $Y$, Roshanpazooh M. Substance-dependent professional drivers in Iran: a descriptive study. Traffic Inj Prev. 2009;10(3):227-230. doi:10.1080/15389580902849017

12. Tavakoli M, Mohammadi L, Yarmohammadi M, Farhoudian A Jafari F, Farhadi MH. Status and Trend of Substance Abuse and Dependence among Iranian Women. Arch Rehabil. 2014;14(5):3037.

13. Momtazi S, Noroozi A, Rawson R. An overview of Iran drug treatment and harm reduction programs. In: el-Guebaly N, Carra G, Galanter M, eds. Textbook of Addiction Treatment: International Perspectives. Milano: Springer; 2015:543-554. doi:10.1007/978-88470-5322-9_25

14. Alam Mehrjerdi Z. Crystal in Iran: methamphetamine or heroin kerack. Daru. 2013;21(1):22. doi:10.1186/2008-2231-21-22

15. Marshall BD, Werb D. Health outcomes associated with methamphetamine use among young people: a systematic review. Addiction. 2010;105(6):991-1002. doi:10.1111/j.13600443.2010.02932.x

16. Meredith CW, Jaffe C, Ang-Lee K, Saxon AJ. Implications of chronic methamphetamine use: a literature review. Harv Rev Psychiatry. 2005;13(3):141-154. doi:10.1080/10673220591003605

17. Scott JC, Woods SP, Matt GE, et al. Neurocognitive effects of methamphetamine: a critical review and meta-analysis. Neuropsychol Rev. 2007;17(3):275-297. doi:10.1007/s11065-0079031-0

18. Farzanegan MR. Effects of international financial and energy sanctions on Iran's informal economy. SAIS Rev Int Aff. 2013;33(1):13-36. doi:10.1353/sais.2013.0008

19. Mokri A, Schottenfeld R. Drug abuse and HIV transmission in Iran-Responding to the public health challenges. In: Celentano DD, Beyrer C, eds. Public health aspects of HIVIAIDS in low and middle income countries. New York, NY: Springer; 2008:583-599. doi:10.1007/978-0-387-72711-0_26

20. Rahimi N, Gozashti MH, Najafipour H, Shokoohi M, Marefati H. Potential effect of opium consumption on controlling diabetes and some cardiovascular risk factors in diabetic patients. Addict Health. 2014;6(1-2):1-6.

21. Geramian N, Akhavan S, Gharaat L, Tehrani AM, Farajzadegan Z. Determinants of drug abuse in high school students and their related knowledge and attitude. J Pak Med Assoc. 2012;62(3 Suppl 2):S62-66

22. Momtazi S, Rawson R. Substance abuse among Iranian high school students. Curr Opin Psychiatry. 2010;23(3):221-226. doi:10.1097/ YCO.0b013e328338630d

23. Rahimi-Madiseh M, Tavakol M, Dennick R. A quantitative study of Iranian nursing students' knowledge and attitudes towards pain: implication for education. Int J Nurs Pract. 2010;16(5):478-483. doi:10.1111/j.1440-172X.2010.01872.x

24. Sharifi H, Shokoohi M, Ahmad RafieiRad A, et al. Methamphetamine Use among Iranian Youth: A Population-based Knowledge, Attitude, and Practice study. Subst Use Misuse. 2017;52(9):1232-1239. doi:1 0.1080/10826084.2017.1303509

25. Stanekzai MR, Todd CS, Orr MG, et al. Baseline assessment of community knowledge and attitudes toward drug use and harm reduction in Kabul, Afghanistan. Drug Alcohol Rev. 2012;31(4):451460. doi:10.1111/j.1465-3362.2011.00352.x

26. Darke S, Hall W, Wodak A, Heather N, Ward J. Development and validation of a multi-dimensional instrument for assessing outcome of treatment among opiate users: the Opiate Treatment Index. $\mathrm{Br} \mathrm{J}$ Addict. 1992;87(5):733-742.

27. Furnham A, Thomson L. Lay theories of heroin addiction. Soc Sci Med. 1996;43(1):29-40.

28. Chesney MA, Barrett DC, Stall R. Histories of substance use and risk behavior: precursors to HIV seroconversion in homosexual 
men. Am J Public Health. 1998;88(1):113-116.

29. Molitor F, Truax SR, Ruiz JD, Sun RK. Association of methamphetamine use during sex with risky sexual behaviors and HIV infection among non-injection drug users. West $J$ Med. 1998;168(2):93-97

30. Bryan A, Moran R, Farrell E, O'Brien M. Drug-related knowledge, attitudes and beliefs in Ireland: report of a nation-wide survey. Dublin: Drug Misuse Research Division, The Health Research Board; 2000

31. World Health Organization (WHO). Substance use in Southern Africa: knowledge, attitudes, practices and opportunities for intervention: summary of baseline assessments in South Africa, the United Republic of Tanzania and Zambia. Geneva: WHO; 2003.

32. Demaio AR, Dugee O, de Courten M, Bygbjerg IC, Enkhtuya P, Meyrowitsch DW. Exploring knowledge, attitudes, and practices related to alcohol in Mongolia: a national population-based survey. BMC Public Health. 2013;13:178. doi:10.1186/1471-2458-13-178

33. Kalebka RR, Bruijns SR, van Hoving DJ. A survey of attitudes towards patient substance abuse and addiction in the Emergency Centre. Afr J Emerg Med. 2013;3(1):10-17. doi:10.1016/j. afjem.2012.09.004

34. Babaie AsI F. Study of Knowledge of Kerman's High School for Boys Students about the Consequences of Psychoactive Substance Abuse. Sci J Hamadan Nurs Midwifery Fac. 2008;16(1):18-28. [Persian]

35. Pourmovahed Z, Yassini Ardakani M, Ahmadieh M, Dehghani $\mathrm{K}$, Kalani Z. Evaluation of the knowledge of rural high school students in Yazd about drugs. J Shahid Sadoughi Univ Med Sci. 2010;18(3):179-183. [Persian].

36. Naderifar M, Ghaljaei F, Akbarizadeh MR, Ebrahimi Tabas E. An investigation in knowledge, attitude and performance of high school students regarding ecstasy abuse, Zahedan-2008. The Quarterly Journal of Fundamentals of Mental Health. 2011;12(4):710-719.

37. Sajjadi M, Shariatifar N, Matlabi M, Abbasnezhad A, Basiri K, Nazemi $\mathrm{H}$. The rate of knowledge and attitude toward psychoactive drugs and its abuse prevalence in Gonabad University students. Horizon Med Sci. 2009;15(2):58-64.

38. Noori R, Daneshmand R, Farhoudian A, Ghaderi S, Aryanfard S, Moradi A. Amphetamine-type stimulants in a group of adults in Tehran, Iran: a rapid situation assessment in twenty-two districts. Iran J Psychiatry Behav Sci. 2016;10(4):1-7. doi:10.17795/ ijpbs-7704

39. Khazaee-Pool M, Moeeni M, Ponnet K, Fallahi A, Jahangiri L, Pashaei T. Perceived barriers to methadone maintenance treatment among Iranian opioid users. Int J Equity Health. 2018;17(1):75. doi:10.1186/s12939-018-0787-z

40. Koohi-Kermani H. History of opium and opium users (Tarikh-eTeriyak va Teriyaki). Tehran: Mohammad Ali Elmi; 1945.

41. Prochaska JO. Transtheoretical model of behavior change. In: Gellman MD, Turner JR, eds. Encyclopedia of behavioral medicine. New York, NY: Springer; 2013:1997-2000. doi:10.1007/978-1-44191005-9

42. Khademi H, Malekzadeh R, Pourshams A, et al. Opium use and mortality in Golestan Cohort Study: prospective cohort study of
50,000 adults in Iran. BMJ. 2012;344:e2502. doi:10.1136/bmj.e2502

43. Ziaaddini $\mathrm{H}$, Ziaaddini MR. The household survey of drug abuse in Kerman, Iran. J Appl Sci. 2005;5(2):380-382.

44. Kheirandish $P$, SeyedAlinaghi $S$, Jahani $M$, et al. Prevalence and correlates of hepatitis $C$ infection among male injection drug users in detention, Tehran, Iran. J Urban Health. 2009;86(6):902-908. doi:10.1007/s11524-009-9393-0

45. Rajabizade G, Ramezani MR, Shakibi MR. Prevalence of opium addiction in Iranian drivers 2001-2003. J Med Sci. 2004;4(3):210213. doi:10.3923/jms.2004.210.213

46. Hamdieh M, Borujerdi A, Motalebi N, Abbasinejad M, Asheri $\mathrm{H}$, Motamedi A. The prevalence of cigarette smoking, alcohol consumption, psychostimulant and cannabinoid drugs abuse among 15 to 35 years old Tehranis. Iran J Psychiatry Behav Sci. 2010;4(2):26-30.

47. Alam-mehrjerdi Z, Mokri A, Dolan K. Methamphetamine use and treatment in Iran: A systematic review from the most populated Persian Gulf country. Asian J Psychiatr. 2015;16:17-25. doi:10.1016/j.ajp.2015.05.036

48. Zolala F, Mahdavian M, Haghdoost AA, Karamouzian M. Pathways to Addiction: A Gender-Based Study on Drug Use in a Triangular Clinic and Drop-in Center, Kerman, Iran. Int J High Risk Behav Addict. 2016;5(2):e22320. doi:10.5812/ijhrba.22320

49. Nemati S, Rafei A, Freedman ND, Fotouhi A, Asgary F, Zendehdel K. Cigarette and Water-Pipe Use in Iran: Geographical Distribution and Time Trends among the Adult Population; A Pooled Analysis of National STEPS Surveys, 2006-2009. Arch Iran Med. 2017;20(5):295-301.

50. Pinkham S, Malinowska-Sempruch K. Women, harm reduction and HIV. Reprod Health Matters. 2008;16(31):168-181. doi:10.1016/ s0968-8080(08)31345-7

51. Meysamie A, Sedaghat M, Mahmoodi M, Ghodsi SM, Eftekhar B. Opium use in a rural area of the Islamic Republic of Iran. East Mediterr Health J. 2009;15(2):425-431.

52. BradyKT, Sinha R. Co-occurring mental and substance use disorders: the neurobiological effects of chronic stress. Am J Psychiatry. 2005;162(8):1483-1493. doi:10.1176/appi.ajp.162.8.1483

53. Elkington KS, Bauermeister JA, Zimmerman MA. Psychological distress, substance use, and HIVISTI risk behaviors among youth. J Youth Adolesc. 2010;39(5):514-527. doi:10.1007/s10964-0109524-7

54. Safizadeh M, Nakhaee N. Causes of increasing trend of divorce in Iranian community: what do the experts think? J Fam Med. 2016;3(4):1064.

55. Haghdoost AA, Baneshi MR, Eybpoosh S, Khajehkazemi R. Comparison of three interview methods on response pattern to sensitive and non-sensitive questions. Iran Red Crescent Med J. 2013;15(6):500-506. doi:10.5812/ircmj.7673

56. Nasirian M, Hosseini Hooshyar S, Haghdoost AA, Karamouzian M. How and Where Do We Ask Sensitive Questions: Self-reporting of STI-associated Symptoms Among the Iranian General Population. Int J Health Policy Manag. 2018;7(8):738-745. doi:10.15171/ ijhpm.2018.18 